\title{
Supported self-management for patients with COPD who have recently been discharged from hospital: a systematic review and meta-analysis
}

This article was published in the following Dove Press journal:

International Journal of COPD

29 April 2015

Number of times this article has been viewed

\author{
Saimma Majothi' \\ Kate Jolly' \\ Nicola R Heneghan ${ }^{2}$ \\ Malcolm J Price \\ Richard D Riley ${ }^{3}$ \\ Alice MTurner ${ }^{4}$ \\ Susan E Bayliss' \\ David J Moore' \\ Sally J Singh ${ }^{5}$ \\ Peymané Adab' \\ David A Fitzmaurice ${ }^{6}$ \\ Rachel E Jordan' \\ 'Public Health, Epidemiology and \\ Biostatistics, School of Health and \\ Population Sciences, University \\ of Birmingham, Birmingham, UK; \\ ${ }^{2} \mathrm{School}$ of Sport, Exercise and \\ Rehabilitation Sciences, University \\ of Birmingham, Birmingham, UK; \\ ${ }^{3}$ Research Institute of Primary Care \\ and Health Sciences, Keele University, \\ Staffordshire, UK; ${ }^{4}$ Queen Elizabeth \\ Hospital Research Laboratories, \\ Birmingham, UK; ${ }^{5}$ Centre for Exercise \\ and Rehabilitation Science, University \\ Hospitals of Leicester NHS Trust, \\ Glenfield Hospital, Leicester, UK; \\ ${ }^{6}$ Primary Care Clinical Sciences, \\ School of Health and Population \\ Sciences, University of Birmingham, \\ Birmingham, UK
}

Correspondence: Rachel E Jordan Public Health, Epidemiology and Biostatistics, School of Health and Population Sciences, Public Health Building, University of Birmingham, Edgbaston, Birmingham BI5 2TT, UK

Tel +44 I2I 4I46775

Email r.e.jordan@bham.ac.uk
Purpose: Although many hospitals promote self-management to chronic obstructive pulmonary disease (COPD) patients post discharge from hospital, the clinical effectiveness of this is unknown. We undertook a systematic review of the evidence as part of a Health Technology Assessment review.

Methods: A comprehensive search strategy with no language restrictions was conducted across relevant databases from inception to May 2012. Randomized controlled trials of patients with COPD, recently discharged from hospital after an acute exacerbation and comparing a self-management intervention with control, usual care or other intervention were included. Study selection, data extraction, and risk of bias assessment were undertaken by two reviewers independently.

Results: Of 13,559 citations, 836 full texts were reviewed with nine randomized controlled trials finally included in quantitative syntheses. Interventions were heterogeneous. Five trials assessed highly supported multi-component interventions and four trials were less supported with fewer contacts with health care professionals and mainly home-based interventions. Total sample size was 1,466 (range 33-464 per trial) with length of follow-up 2-12 months. Trials varied in quality; poor patient follow-up and poor reporting was common. No evidence of effect in favor of self-management support was observed for all-cause mortality (pooled hazard ratio $=1.07 ; 95 \%$ confidence interval $[0.74$ to 1.55$] ; I^{2}=0.0 \%,[\mathrm{n}=5$ trials] $)$. No clear evidence of effect on all-cause hospital admissions was observed (hazard ratio 0.88 [0.61, 1.27] $I^{2}=66.0 \%$ ). Improvements in St George's Respiratory Questionnaire score were seen in favor of self-management interventions (mean difference $=3.84$ [1.29 to 6.40$] ; I^{2}=14.6 \%$ ), although patient follow-up rates were low.

Conclusion: There is insufficient evidence to support self-management interventions postdischarge. There is a need for good quality primary research to identify effective approaches. Keywords: chronic obstructive pulmonary disease, self-management support, post-discharge, systematic review

\section{Introduction}

Chronic obstructive pulmonary disease (COPD) is a progressive and non-reversible lung disease characterized by breathlessness and chronic productive cough. ${ }^{1}$ Many patients suffer exacerbations requiring hospital admission, at high cost to the health care system worldwide and contributing to poorer prognosis for patients. ${ }^{1}$

Approximately $30 \%$ of patients admitted to hospital for an exacerbation of COPD are readmitted within 3 months following discharge ${ }^{2}$ and thus form a particularly high-risk group with significant potential to benefit. A recent systematic review has shown that supported self-management interventions can be effective in reducing 
future respiratory admissions among stable COPD patients, ${ }^{3}$ although simple self-management education without support is not effective. ${ }^{4}$ Some hospitals already promote selfmanagement to patients as a component of discharge care after admission for severe exacerbation, ${ }^{5}$ but it is not yet clear whether it is effective at this time point.

Self-management for patients with COPD is complex and challenging. ${ }^{6}$ It requires patients to be able to manage various facets of their condition on a daily basis, including understanding and taking their medications appropriately with good inhaler technique, early recognition of exacerbations of symptoms and early instigation of treatment during an exacerbation, receiving annual influenza vaccinations, managing their breathlessness (including stress management/ relaxation) to allow them to undertake activities of daily living, bronchial clearance techniques, taking regular exercise to maintain their lung function and exercise capacity, quitting smoking, and maintaining a healthy diet. ${ }^{7-9}$ In reality, the true extent to which patients manage these aspects is not well described, but likely to be sub-optimal. Patients report being able to recognize the onset of exacerbations with both clinically-recognized and experiential symptoms and signs, and that they wanted to self-manage, but did not always want to take antibiotics or steroids and might delay contacting health care professionals until in a crisis. ${ }^{10}$

Self-management support for COPD is less well developed than in other long-term conditions. A variety of tools are available, such as the "Living well with COPD" program developed by the Montreal Chest Institute, which was first shown to be effective in $2003,{ }^{11}$ but no one consistent recommended approach. ${ }^{12}$ Furthermore, there is considerable overlap between programs which are defined as self-management and other more complex supervised programs such pulmonary rehabilitation, integrated care or case management. A continuum of support is now recognized which should ideally be personalized to reflect an individual patient's needs, including disease severity and other co-morbidities..$^{9,13}$ Exacerbations result in marked increases in both physical and emotional distress for patients, taking several weeks to recover from. ${ }^{14}$ Therefore interventions effective in a stable state may not be appropriate after patients have recently experienced an exacerbation.

We therefore report a systematic review undertaken to assess the clinical effectiveness of interventions to support self-management among patients with COPD who have recently been discharged from hospital following an acute exacerbation. Self-management is not universally defined, however for the purposes of this review, self-management is defined as the "ability of a patient to deal with all that a chronic disease entails, including symptoms, treatment, physical and social consequences and lifestyle changes", ${ }^{15}$ and we therefore included interventions which supported any component of self-management, comparing against usual care, control or other self-management intervention.

\section{Methods}

This was a protocol driven systematic review registered with PROSPERO (reference number CRD42011001588), and undertaken as part of a wider Health Technology Assessment (HTA) review.

\section{Search strategy}

The following databases were searched from inception to May 2012 with no language restrictions: MEDLINE (Ovid), MEDLINE In Process (Ovid), EMBASE (Ovid), Cochrane (Wiley) Central Register of Controlled Trials (CENTRAL), Science Citation Index (ISI), PEDro physiotherapy evidence database, PsycINFO (Ovid), and the Cochrane Airways specialized register. Search terms for respiratory disorders such as "COPD" and "pulmonary emphysema" were combined with terms for self-management and post-discharge management such as "self management", "action planning", and "discharge planning" in a sensitive search strategy. Citation lists of all included studies and relevant systematic reviews were scanned.

\section{Study selection}

Two reviewers independently screened titles, abstracts, and full texts of any relevant papers using pre-defined inclusion and exclusion criteria. We sought randomized controlled trials (RCTs) which recruited patients with moderate to severe COPD (defined clinically \pm spirometry), at the point of discharge or within 6 weeks following hospitalization for an acute exacerbation of COPD. Any self-management interventions were eligible which included one or more components commonly included in self-management interventions, such as action plans, exercise, education, inhaler technique, bronchial hygiene and breathing techniques, stress management and relaxation, nutritional programs, patient empowerment, support groups and telecare (Table S1), provided in either hospital or community setting with a usual care, control, sham intervention or other self-management intervention comparator. Primary outcomes of interest were health service utilization (primary care consultations, hospital admissions/ re-admissions, duration of admissions, emergency department [ED] visits) and mortality. Any studies with important 
relevant secondary outcomes such as exacerbations, health related quality of life (HRQoL), anxiety, depression, dyspnea, self-efficacy, lung function, and specific behaviors such as exercise/physical activity were also included.

Studies were excluded if the patient population was mixed unless approximately $90 \%$ of the total sample had COPD or data for COPD were presented and analyzed separately.

Trials of pulmonary rehabilitation were excluded as the effects of early pulmonary rehabilitation have been reviewed previously. ${ }^{16}$ Trials of smoking cessation alone were also excluded as smoking cessation has established clinical effectiveness. Interventions based exclusively on case-management, integrated care, disease management and hospital at home were excluded unless components of selfmanagement were part of the intervention.

\section{Data extraction and assessment of risk of bias}

Data extraction and quality assessment were independently undertaken by two reviewers with a third reviewer overseeing and resolving any disagreements. Quality assessment was undertaken using the Cochrane Risk of Bias tool. ${ }^{17}$

\section{Data synthesis}

Interventions were placed into natural groupings decided before inspection of the results and agreed with clinical experts:

1. more supported (six or more contacts, or 6 or more weeks in duration),

2 . less supported (fewer than six contacts or fewer than 6 weeks in duration).

Meta-analyses were performed using a random effects model where studies were judged appropriately homogeneous. Heterogeneity was assessed using the $I^{2}$ statistic. All continuous data were presented using a mean difference with 95\% confidence intervals (CIs) and time to event outcomes were presented as hazard ratios (HRs) to account for varying lengths of follow-up. Follow-up time was converted to weeks. The results of the St George's Respiratory Questionnaire (SGRQ) were multiplied by -1 so that positive differences equated to improved HRQoL. Where HRs were not reported, methods by Parmar et $\mathrm{al}^{18}$ and Perneger ${ }^{19}$ were used. Rates of admissions and ED visits (ie, where patients could have multiple events) were calculated under the assumption that all patients in both arms were followed up for the duration of the study and that the rates were constant over time. All data analyses were undertaken using STATA 12 (StataCorp LP, College Station, TX, USA).

\section{Results Search results}

Of 13,559 identified records, 836 full papers were retrieved and ten RCTs (including one cluster RCT) ${ }^{20}$ reported in 12 papers met the final inclusion criteria (Figure 1). ${ }^{20-31}$ However, one included trial of 46 patients $^{21,22}$ reported an exercise-only intervention which was more similar in nature to pulmonary rehabilitation and quite different to the other studies; therefore after consideration was not reported in any of the outcome analyses below (see Table S2 for full details).

\section{Characteristics of included trials}

The remaining nine RCTs ranged in size from $33^{25}$ to $464^{23}$

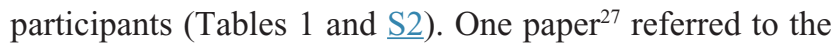
Spanish center of a European study. ${ }^{24}$ Participants were recruited in hospital during an exacerbation of COPD or at (or immediately after) discharge. All studies were set among patients living at home except for the cluster RCT in nursing homes. ${ }^{20}$

Inclusion was usually based on a clinical diagnosis of COPD, except the most recent study which also required patients to meet spirometric criteria for airflow obstruction. ${ }^{23}$ One study included a mixed population of patients with chronic lung disease. ${ }^{30}$

Mean age of participants was similar across the included RCTs (67-75 years), except in the trial set in nursing homes where the mean age was approximately 80 years. ${ }^{20}$ Sex distribution was variable across studies ( $37 \%$ to $97 \%$ males). Where reported, severity of disease was consistent with mean forced expiratory volume in one second $\left(\mathrm{FEV}_{1}\right)$ ranging from approximately $31 \%$ to $42 \%$ of predicted values. Most patients were described as having moderate or severe COPD.

Interventions were varied and all contained several selfmanagement components. All were compared against usual care (Tables 1 and $\underline{\text { S2}}$, Figure 2). Five trials were classified as more supported, with several face-to-face visits and interventions lasting 6 weeks or more, and follow-up for 6-12 months. ${ }^{20,23-25,27,30}$ The largest and most recent trial was described as a supported self-management intervention, and used the "Living Well with COPD" program, a comprehensive 12 months education and behavior-change package with motivation and support by nurses trained in self-regulation theory, and including medications to commence at the onset of an exacerbation. ${ }^{23}$ Two other trials had a similar level of support and similar interventions to each other: a 12 months trial of integrated care ${ }^{24,27}$ and a 6 months trial of community nurse-support. ${ }^{30}$ One other trial comprised several visits to an outpatient respiratory nurse/chest physician 


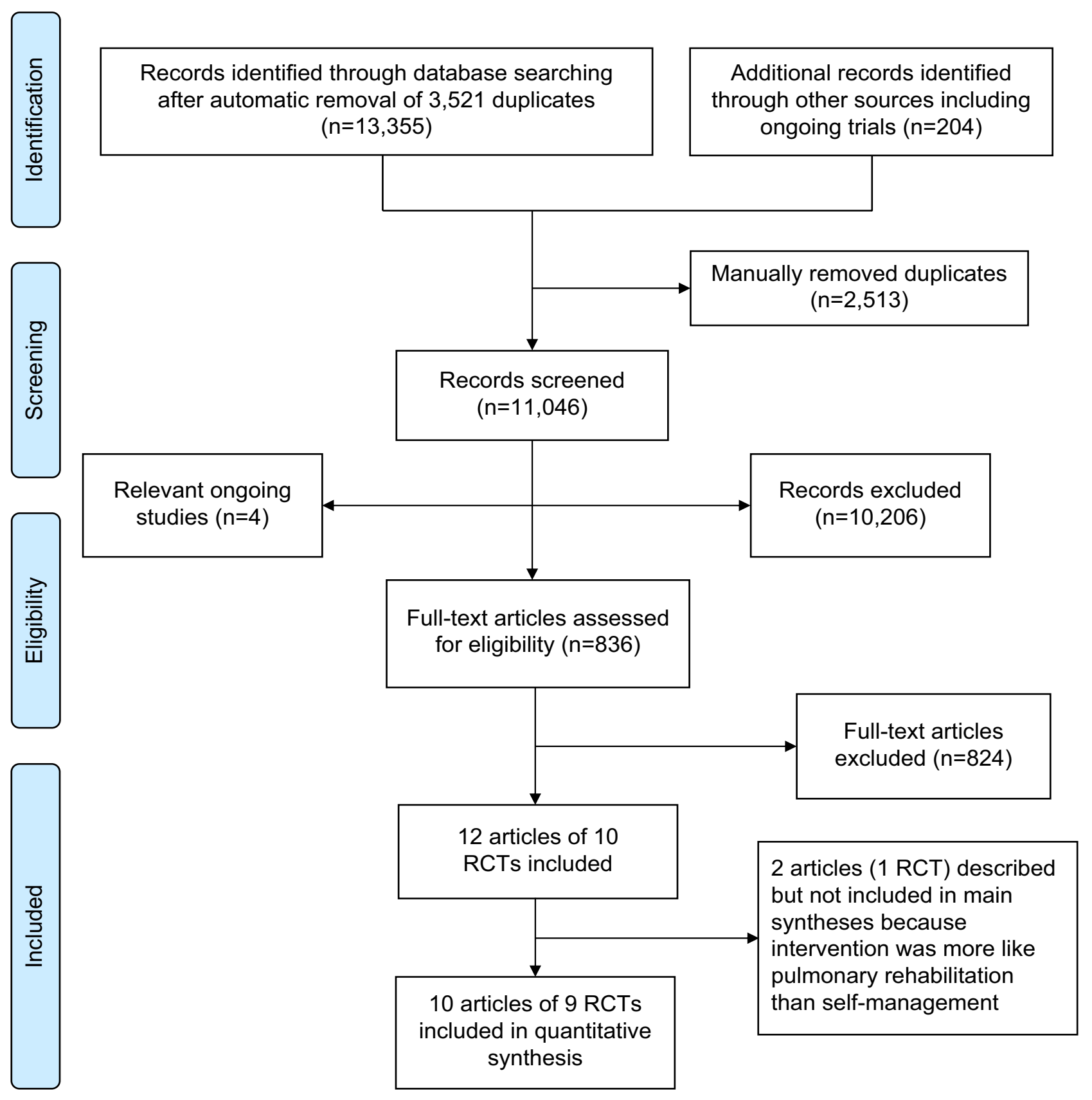

Figure I Flow diagram summarizing the selection process for clinical-effectiveness studies. Abbreviation: RCT, randomized controlled trial.

over 6 months, ${ }^{25}$ and the final trial in the "most-supported" category provided care and support to both patients and staff of nursing homes. ${ }^{20}$

Four trials had fewer contacts and 2-3 months follow-up. These interventions included two trials of home-based self-management visits ${ }^{28,29}$ covering a comprehensive range of components; case-management with telephone follow-up ${ }^{26}$ and a telephonebased intervention to help patients manage dyspnea. ${ }^{31}$

All interventions had at least two components and most were multi-component. All included training on medication adherence; five trials inhaler technique, five trials smoking cessation, six trials nutritional advice, seven trials promoted exercise, and four trials management of dyspnea. Five trials discussed early recognition of exacerbations but only one trial provided patients with medications to self-treat exacerbations. ${ }^{23}$ Only two trials cited behavior change theories upon which the interventions were based. ${ }^{23,31}$ Most interventions commenced after discharge although three trials included a review of needs and brief initial education session before discharge. ${ }^{26,29,30}$ 


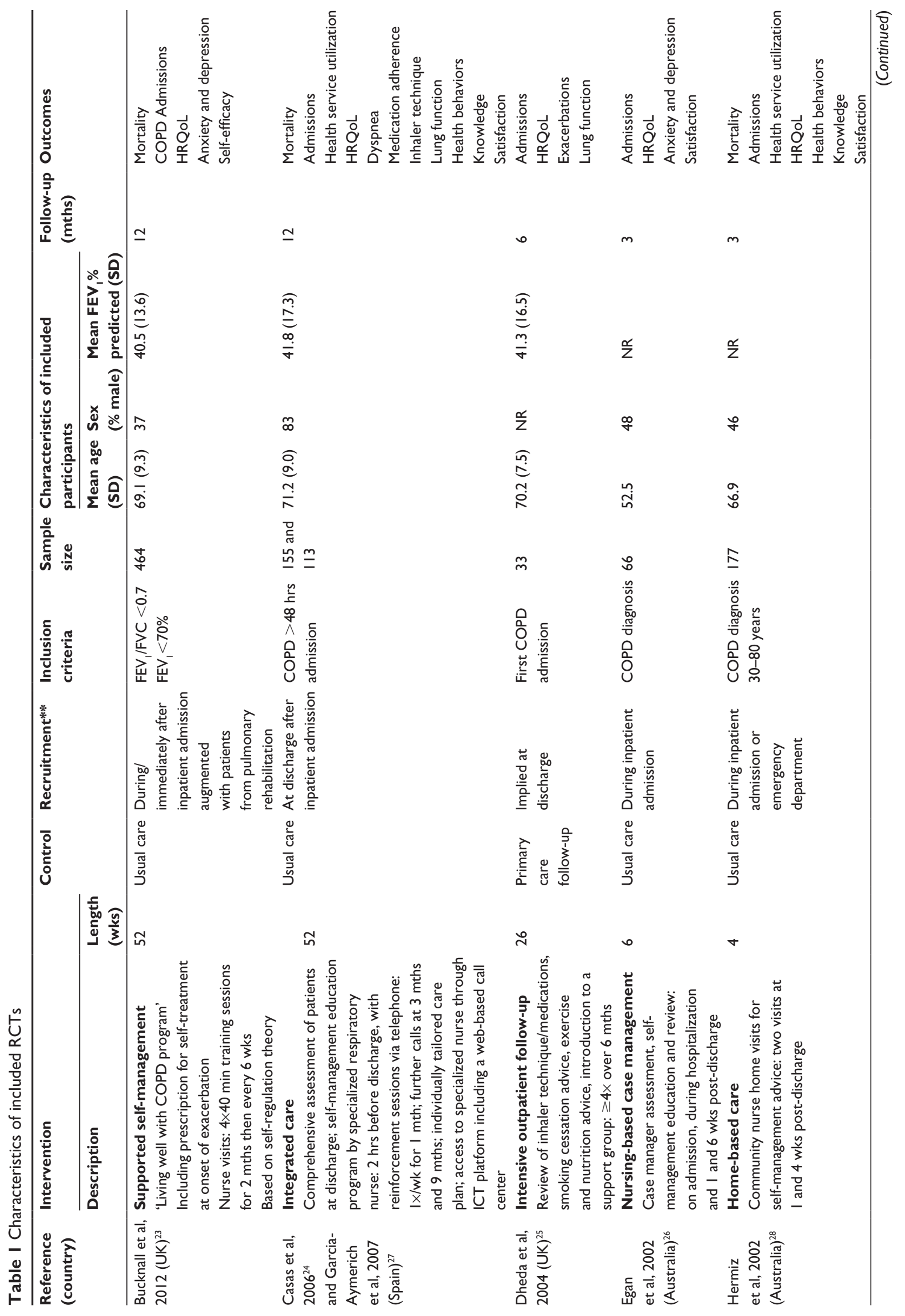




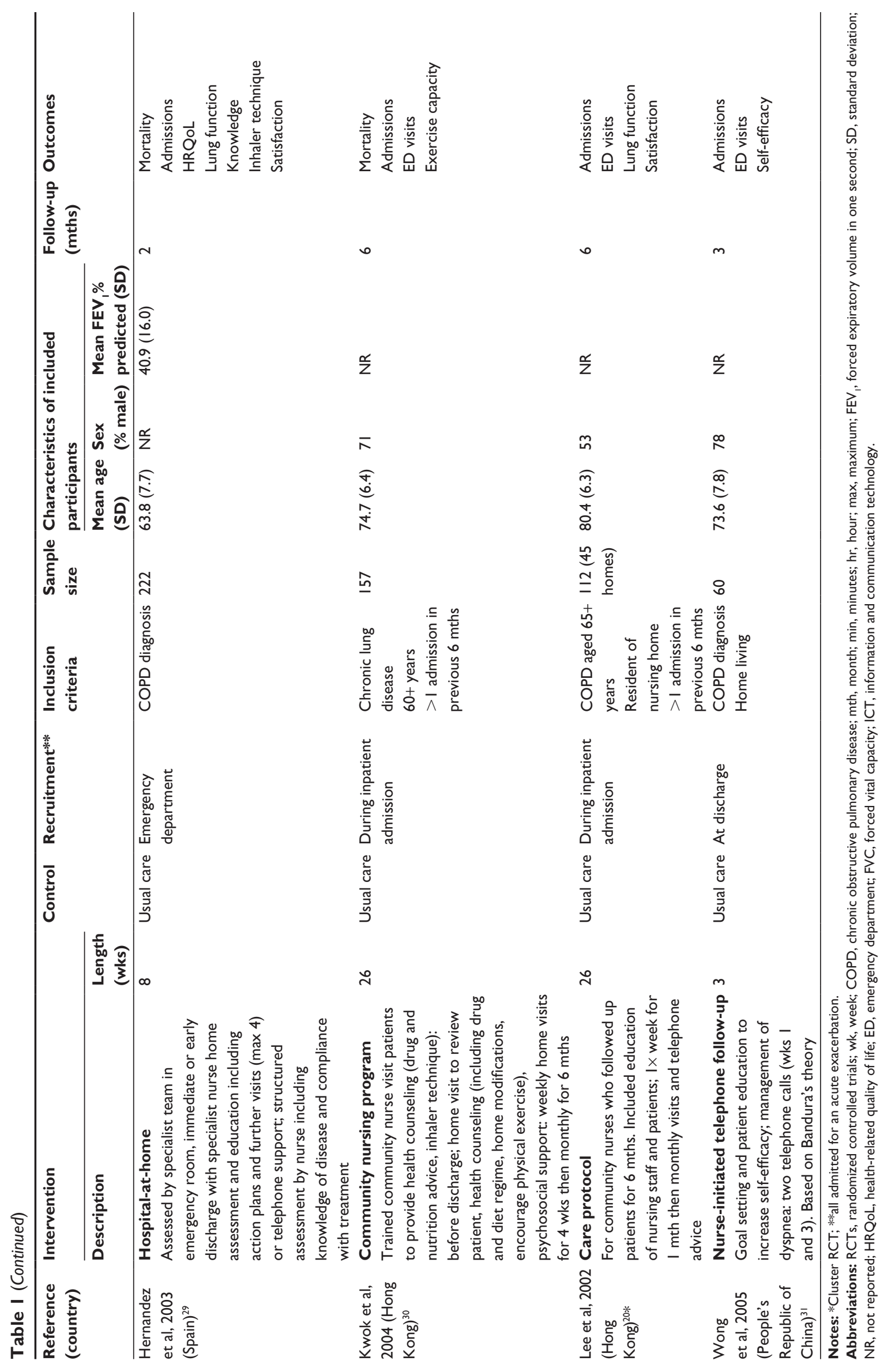


More supported

$(\geq 6$ contacts or unspecified
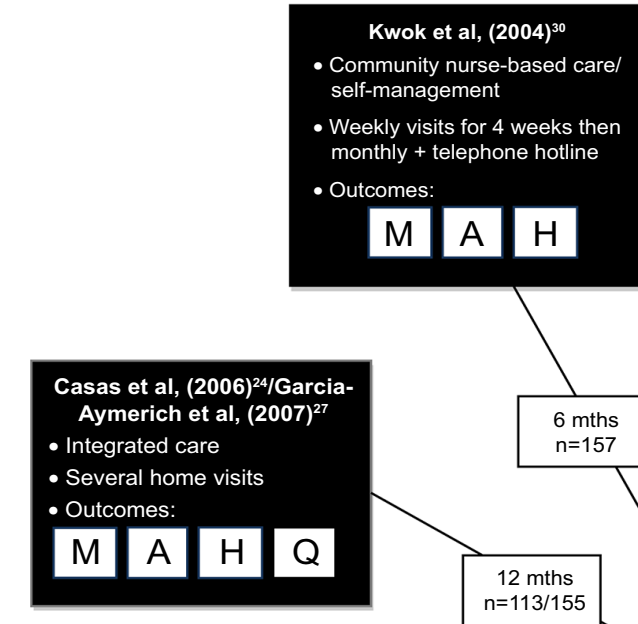

\section{Kwok et al, (2004) $)^{30}$} mmunity nurse-based care/ telephone hotline
Dheda et al, (2004) ${ }^{25}$ - Visits to respiratory

nurse/physician $\geq 4$

times

times

- Outcomes:

\section{A $Q$}

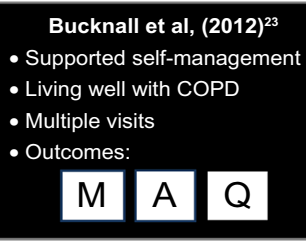

- Living well with COPD

Multiple visits

\begin{tabular}{l|l|l}
$M$ & $A$ & $Q$ \\
\hline
\end{tabular}

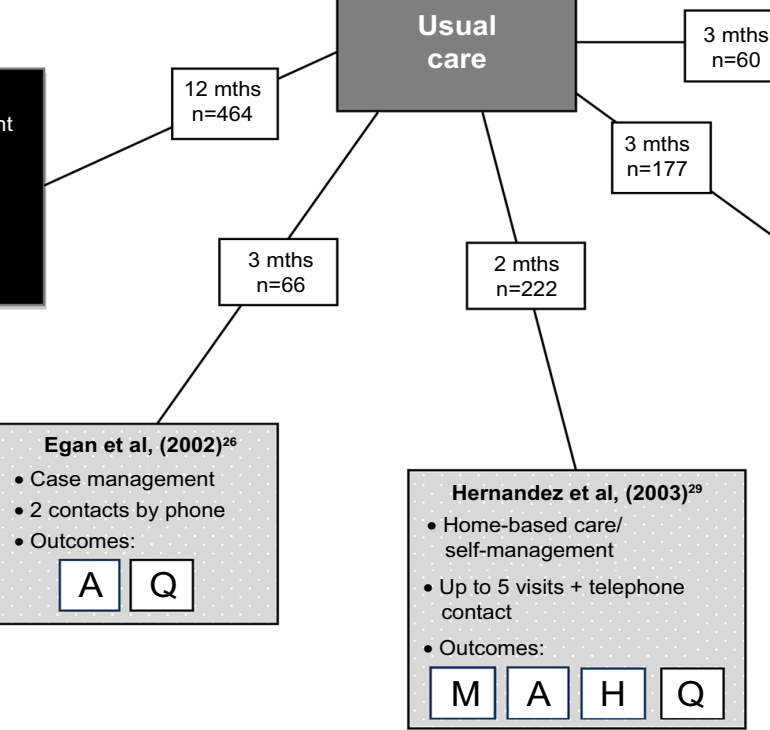

Lee et al, (2002)

- Care and self-management

support to nursing home

staff/patients

- $4 \times$ weekly visits, then monthly

- Outcomes

\section{A $\mathrm{H}$}

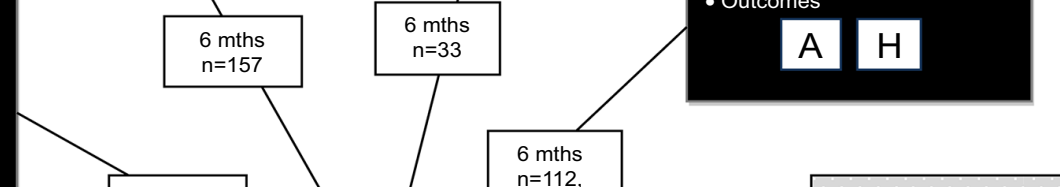

Wong et al, (2005) $)^{31}$

- Telephone-based selfmanagement of dyspnea

- 2 contacts

- Outcomes:

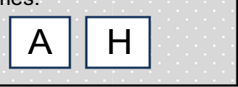

Hermiz et al, (2002) ${ }^{28}$

- Home-based care

self-management

- 2 visits

- Outcomes:

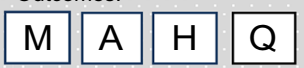

Hernandez et al, (2003)

Home-based care

p to 5 visits + telephone

$M \quad A \quad \mathrm{Q}$

Less supported ( $<6$ contacts or unspecified contacts/ $<6$ weeks duration)

Figure 2 Summary diagram of interventions.

Abbreviations: COPD, chronic obstructive pulmonary disease; $m$ th, month.

\section{Risk of bias}

The quality of reporting and conduct of the included studies was often low, especially among the smaller, older studies (Table S3). Appropriate methods of randomization were used in six trials, although methods were unclear in the remaining three. ${ }^{20,25,28}$ Allocation concealment was insufficiently described in all except the most recent trial. ${ }^{23}$

Blinding was not common for self-management interventions therefore many patient-reported outcomes such as quality of life were subject to high risk of detection bias. Health care utilization outcomes, eg, hospital admissions and mortality related data were judged less likely to be affected.
Reporting and analyses of results was unclear or incorrect in four of the older trials. ${ }^{20,25,26,29}$ The clustering was not accounted for in the analysis of the cluster trial, ${ }^{20}$ which, although having small numbers of patients per cluster, would possibly underestimate the uncertainty in the effect size.

The most obvious flaw was the lack of completeness in follow-up for clinical measures and especially self-reported questionnaire outcomes, which was considerably less than $70 \%$ in some trial arms. ${ }^{23,25}$ One trial gave no table of characteristics, ${ }^{15}$ thus weakening ability to assess baseline imbalance. 


\section{Effectiveness results}

\section{Primary outcomes}

Mortality

Five trials contributed mortality data to the analysis (Figure 3). ${ }^{23,24,28-30}$ There was a wide range of event rates across the trials. Despite the general heterogeneity of interventions, there was no statistical heterogeneity and overall no evidence of effect on all-cause mortality (HR 1.07 [95\% CI $0.74,1.55]$. $\left(I^{2}=0.0 \%\right)$. Only one trial reported COPD-specific mortality therefore this outcome could not be explored. ${ }^{23}$

\section{Hospital admissions}

There were six trials with data which could be combined to assess the overall effect on time to first admission (Figure 4). ${ }^{23-25,28-30}$ These were all-cause admissions except for the most recent trial which provided COPD-specific admissions. Overall, statistical heterogeneity was high $\left(I^{2}=66.0 \%\right)$, and sub-dividing by level of support explained little of this. One of the studies which may have contributed to the remaining heterogeneity in the non-exercise-based studies is the small study of 33 participants by Dheda et $\mathrm{al}^{25}$ which was poorly reported, had signs of inadequate randomization, and very high loss-to-follow-up, especially in the intervention arm. This study had the most extreme results in its category. The remaining studies were overall of moderate-good quality. Overall there was no clear evidence of effect (HR 0.88 [0.61, 1.27]). A similar general lack of effect was observed when evaluating hospital admission rates (igure S1) (five trials).

\section{ED visits and general practitioner consultations}

Four trials reported mean ED visits per patient ${ }^{20,29-31}$ and two reported first visit. ${ }^{28,29}$ Although two of the three shorter trials suggested potential reduction in ED visits, the two trials with a longer follow-up of 6 months failed to demonstrate any evidence of an effect (Figure S2) ${ }^{20,30}$ Similarly, no differences were observed in physician contacts reported between self-management interventions and usual care (Table 2)..$^{24,28}$

\section{Secondary outcomes}

Exacerbations were inadequately reported..$^{25}$ Five trials measured HRQoL using two different scales..$^{23,25,27,29}$ Selfmanagement interventions resulted in an improvement of 3.84 points $(95 \%$ CI $1.29,6.40)$ on the SGRQ scale compared with control (Figure 5); however follow-up ranged from $\sim 25 \%$ to $83 \%$ across studies therefore this finding should be treated with caution. The small study showing the most extreme results suffered from poor reporting and likely bias. ${ }^{25}$ No overall effect was observed among trials reporting the EuroQol 5D, a generic quality of life tool (Figure S3).

Although there were data on less than half of the sample, in one trial the intervention group had a mean reduction of 1.06 points $(95 \%$ CI $0.04,2.08)$ in the Hospital Anxiety and Depression Score anxiety score, ${ }^{23}$ and another trial demonstrated a mean reduction of 1.5 points $(95 \%$ CI $0.62,2.38)$ in the Anxiety and Insomnia component of the General Health Questionnaire relative to control (Table 2). ${ }^{20}$ This trial also showed a reduction in depression score (mean difference -1.0 [-1.97, -0.03]), although follow-up rates were not reported and the CIs may be underestimated as adjustment for clustering was not possible. ${ }^{20}$ There was no evidence of such effect in the larger trial. ${ }^{23}$

Exercise capacity was assessed in the trial of nursesupported discharge in Hong Kong where exercise prescribed by the physiotherapist was encouraged as part of the intervention. ${ }^{30}$ Having retained nearly $90 \%$ of participants, it reported no difference after 6 months (mean difference in 6 minute walk distance $24 \mathrm{~m}[95 \% \mathrm{CI}-7.1,55.1 \mathrm{~m}]),{ }^{20}$ although the substantial baseline imbalance was not taken into account.

No evidence of effect on dyspnea was observed in the only trial (of integrated care) which evaluated it. ${ }^{27}$ No evidence of improvement in lung function was observed in any of the trials. ${ }^{20,27,29}$ Three trials reported significantly better knowledge and ability to recognize and treat exacerbations among patients receiving the self-management intervention, ${ }^{27-29}$ although results were inconsistent for reported self-efficacy (Table 3).23,31 Two trials reported significantly better adherence to inhaler treatment and inhaler technique; ${ }^{27,29}$ however, this was not matched by improvements in smoking behaviors or uptake of vaccines. Effects on physical activity were inconsistent. ${ }^{27,29}$

\section{Discussion}

\section{Key results}

Despite a rigorous search we only identified ten $\mathrm{RCTs} \mathrm{s}^{20-31}$ which evaluated the effectiveness of interventions providing self-management support to patients shortly after discharge from hospital with an acute exacerbation of their COPD, and one of these was better classified as pulmonary rehabilitation. ${ }^{21,22}$ Few of the trials had consistently low risk of bias. Some older studies in particular were small and suffered from inadequate reporting and high loss-to-follow-up, particularly affecting patient-reported outcomes such as HRQoL.

There was no apparent evidence of effect of self-management interventions on all-cause mortality. 


\section{Mortality: all cause}

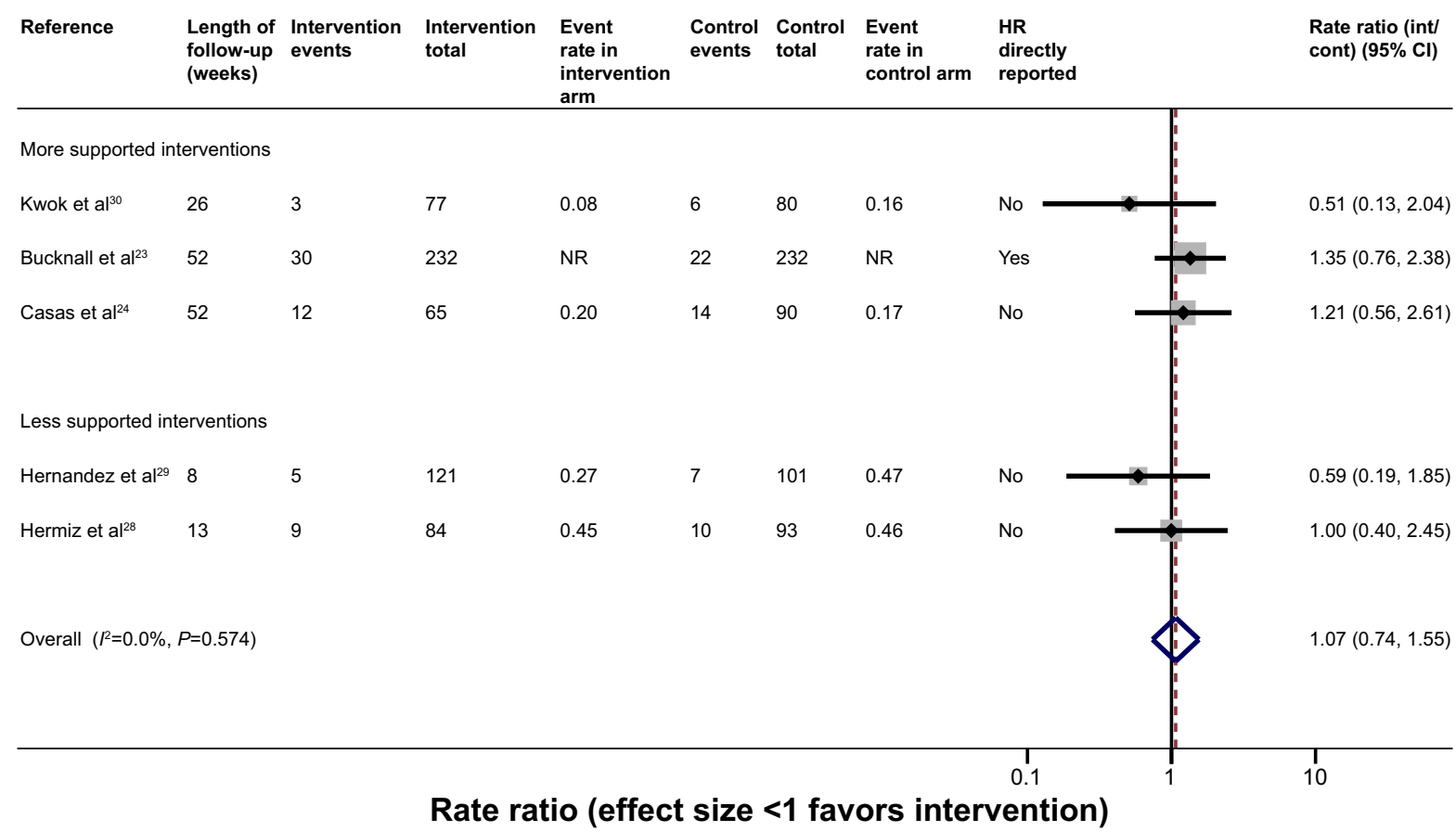

Figure 3 Effect of self-management support interventions on mortality.

Abbreviations: $\mathrm{Cl}$, confidence interval; NR, not reported; HR, hazard ratio; int, intervention; cont, control.

\section{Time to first admission}

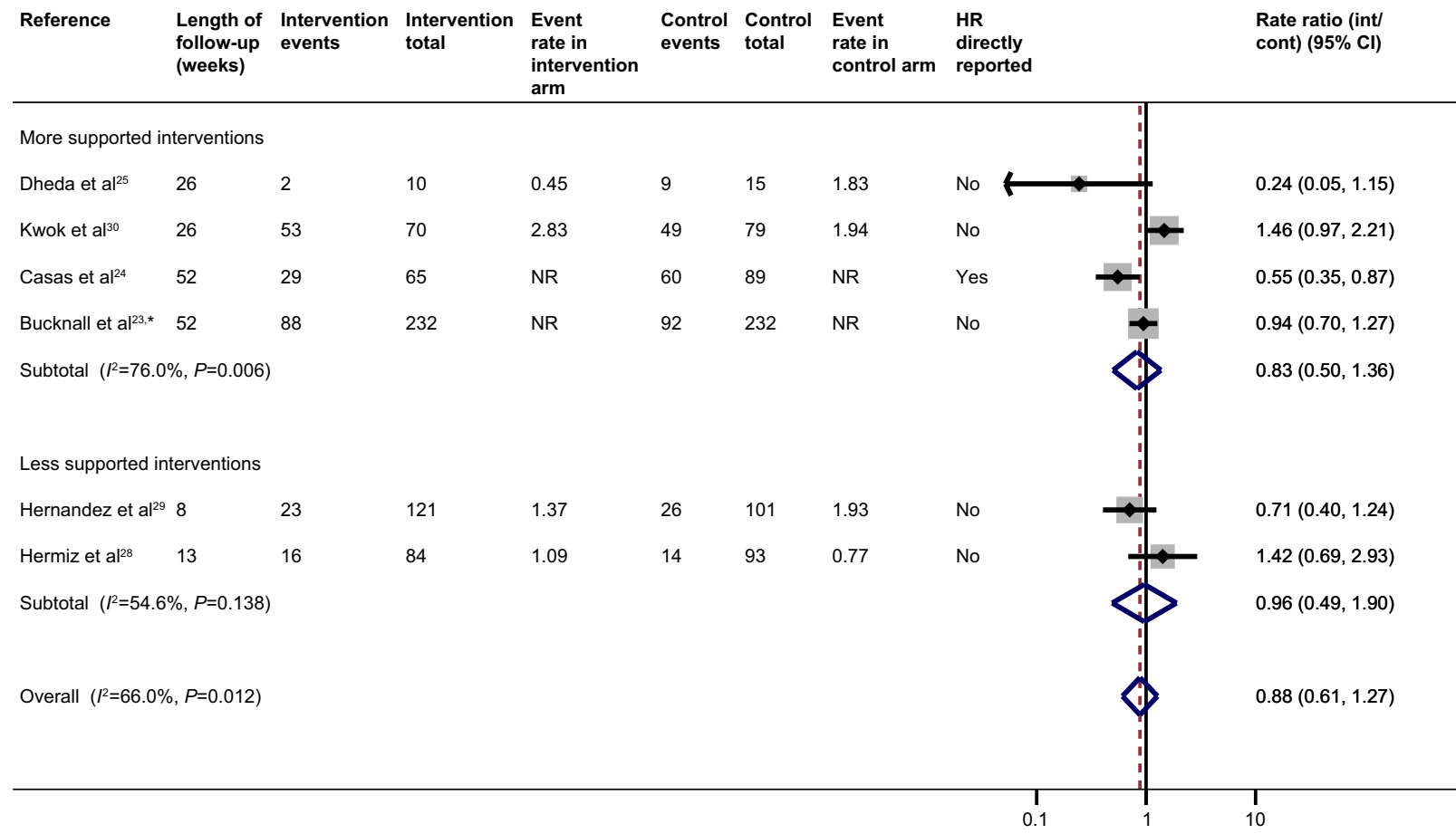

Rate ratio (effect size $<1$ favors intervention)

Figure 4 Effect of self-management support interventions on time to first re-admission.

Notes: *COPD-related admission. All others unknown/unclear cause.

Abbreviations: COPD, chronic obstructive pulmonary disease; NR, not reported; HR, hazard ratio; $\mathrm{Cl}$, confidence interval; int, intervention; cont, control. 


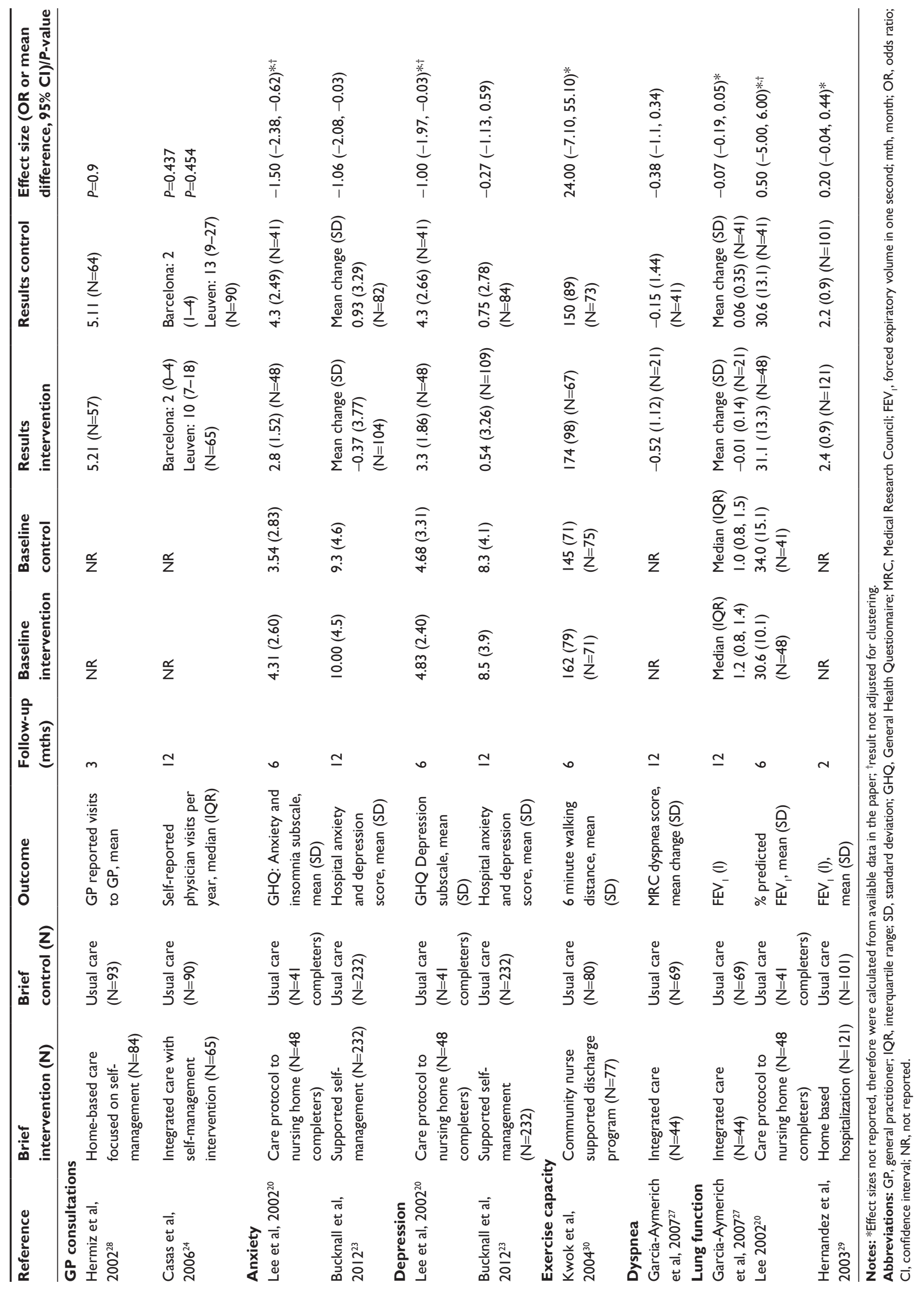




\begin{tabular}{|c|c|c|c|c|c|c|c|c|}
\hline \multirow[b]{2}{*}{ Reference } & \multicolumn{7}{|c|}{ Quality of life: SGRQ } & \multirow[b]{2}{*}{$\begin{array}{l}\text { Mean difference } \\
\text { (int-cont) }(95 \% \mathrm{Cl})\end{array}$} \\
\hline & $\begin{array}{l}\text { Length of } \\
\text { follow-up } \\
\text { (weeks) }\end{array}$ & $\begin{array}{l}\text { Baseline } \\
\text { difference } \\
\text { (int-cont) }\end{array}$ & $\begin{array}{l}\text { Analysis } \\
\text { method }\end{array}$ & $\begin{array}{l}\text { Follow-up in } \\
\text { intervention arm } \\
\mathrm{n} \text { (end)/n (start) (\%) }\end{array}$ & $\begin{array}{l}\text { Follow-up in } \\
\text { control arm } \\
\mathrm{n} \text { (end)/n (start) (\%) }\end{array}$ & $\begin{array}{l}\text { Intervention } \\
\text { category }\end{array}$ & & \\
\hline Dheda et $\mathrm{a}^{25}$ & 26 & NR & $\begin{array}{l}\text { Comparison of } \\
\text { final scores }\end{array}$ & $10 / 15(67 \%)$ & $15 / 18(83 \%)$ & $\begin{array}{l}\text { More supported } \\
\text { interventions }\end{array}$ & $\rightarrow$ & $15.00(2.46,27.54)$ \\
\hline $\begin{array}{l}\text { Garcia- } \\
\text { Aymerich et al2 }\end{array}$ & 52 & -9.3 & $\begin{array}{l}\text { Comparison of } \\
\text { change since baseline }\end{array}$ & $21 / 44(48 \%)$ & $41 / 69(59 \%)$ & $\begin{array}{l}\text { More supported } \\
\text { interventions }\end{array}$ & & $2.39(-5.78,10.56)$ \\
\hline Bucknall et a ${ }^{23}$ & 52 & 0.8 & ANCOVA & $69 / 232(30 \%)$ & $53 / 232(23 \%)$ & $\begin{array}{l}\text { More supported } \\
\text { interventions }\end{array}$ & 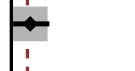 & $4.52(-0.03,9.07)$ \\
\hline Hernandez et al ${ }^{29}$ & 8 & NR & $\begin{array}{l}\text { Comparison of } \\
\text { change since baseline }\end{array}$ & NR/121 (NR) & NR/101 (NR) & $\begin{array}{l}\text { Less supported } \\
\text { interventions }\end{array}$ & $\rightarrow$ & $4.50(0.66,8.34)$ \\
\hline Hermiz et al ${ }^{28}$ & 13 & 3.02 & $\begin{array}{l}\text { Comparison of } \\
\text { change since baseline }\end{array}$ & $67 / 84(80 \%)$ & $80 / 93(86 \%)$ & $\begin{array}{l}\text { Less supported } \\
\text { interventions }\end{array}$ & $\rightarrow$ & $1.32(-2.97,5.61)$ \\
\hline Overall $\quad I^{2}=14.6 \%$, & $P=0.321)$ & & & & & & (1) & $3.84(1.29,6.40)$ \\
\hline
\end{tabular}

\section{Mean difference in SGRQ (effect size $>0$ favors intervention)}

Figure 5 Effect of self-management support interventions on health-related quality of life (SGRQ score).

Note: Positive effect signifies improvement.

Abbreviations: SGRQ, St George's Respiratory Questionnaire; NR, not reported; ANCOVA, analysis of covariance; int, intervention; cont, control; Cl, confidence interval.

For all-cause hospital admission, although the direction of effect weakly favored the self-management intervention, there was substantial uncertainty and thus no clear evidence of an effect. Despite sub-dividing by the level of support provided in the intervention, we were unable to explain the heterogeneity observed. It is possible however, that the effects on re-admissions would be diluted because we extracted admissions due to any cause (although where reported the majority were for respiratory causes).

In terms of health outcomes, the most consistent effects were observed on patients' quality of life, with an overall improvement of 3.8 points in the SGRQ score (close to the minimally clinically important difference of four points). ${ }^{32}$ This estimate should however be treated with caution because although it is unlikely to be due to chance, there were substantial and differential losses-to-follow-up between intervention and usual care which could bias the results in favor of a positive effect. Indeed, the authors of the largest trial indicated that the results from their trial could be unreliable. ${ }^{23}$ The reduction in anxiety exhibited in two trials supports some possible improvements up to 1 year, although the effect observed was small and should be treated with caution. ${ }^{20,23}$

Overall, although knowledge about COPD improved and patients reported better adherence to medications and more effective use of inhalers, there was limited evidence of effect on health related behaviors or on self-efficacy.

\section{How this fits into other literature}

This systematic review addresses the effectiveness of selfmanagement support provided to COPD patients soon after hospital discharge.

The majority of the studies and reviews of self-management support are set among patients who have COPD in a stable state. A systematic review of self-management interventions showed a reduction in respiratory admissions (odds ratio [OR] $0.57[0.43,0.75]$ [ $\mathrm{n}=9$ trials]), ${ }^{3}$ although no significant effect on all-cause admissions (OR 0.77 [0.45, $1.30]$ ), or on all-cause mortality (OR $0.79,95 \%$ CI 0.58 to 1.07); but a mean improvement of $3.51(1.65,5.37)$ points on the SGRQ score ( $\mathrm{n}=10$ trials). A review of integrated disease management found a similar improvement in HRQoL: SGRQ 3.71 points $(1.6,5.8)(n=13)$; chronic respiratory disease questionnaire $1.02(0.67,1.36)(\mathrm{n}=4)$ and respiratory admissions (OR 0.68 [0.47, 0.99] [n=7]), and a similar lack of effect on mortality. ${ }^{33}$ Conversely, a review of action plans alone found little evidence of benefit on HRQoL or health care utilization. ${ }^{4}$ Our results generally showed weaker evidence of effects on all-cause admissions and mortality, although the lower bound of CIs from our review are consistent with their results. We did not have sufficient data to measure effects on respiratory admissions or effects of action plans alone. The effect we observed on HRQoL however, was very similar to these reviews. 


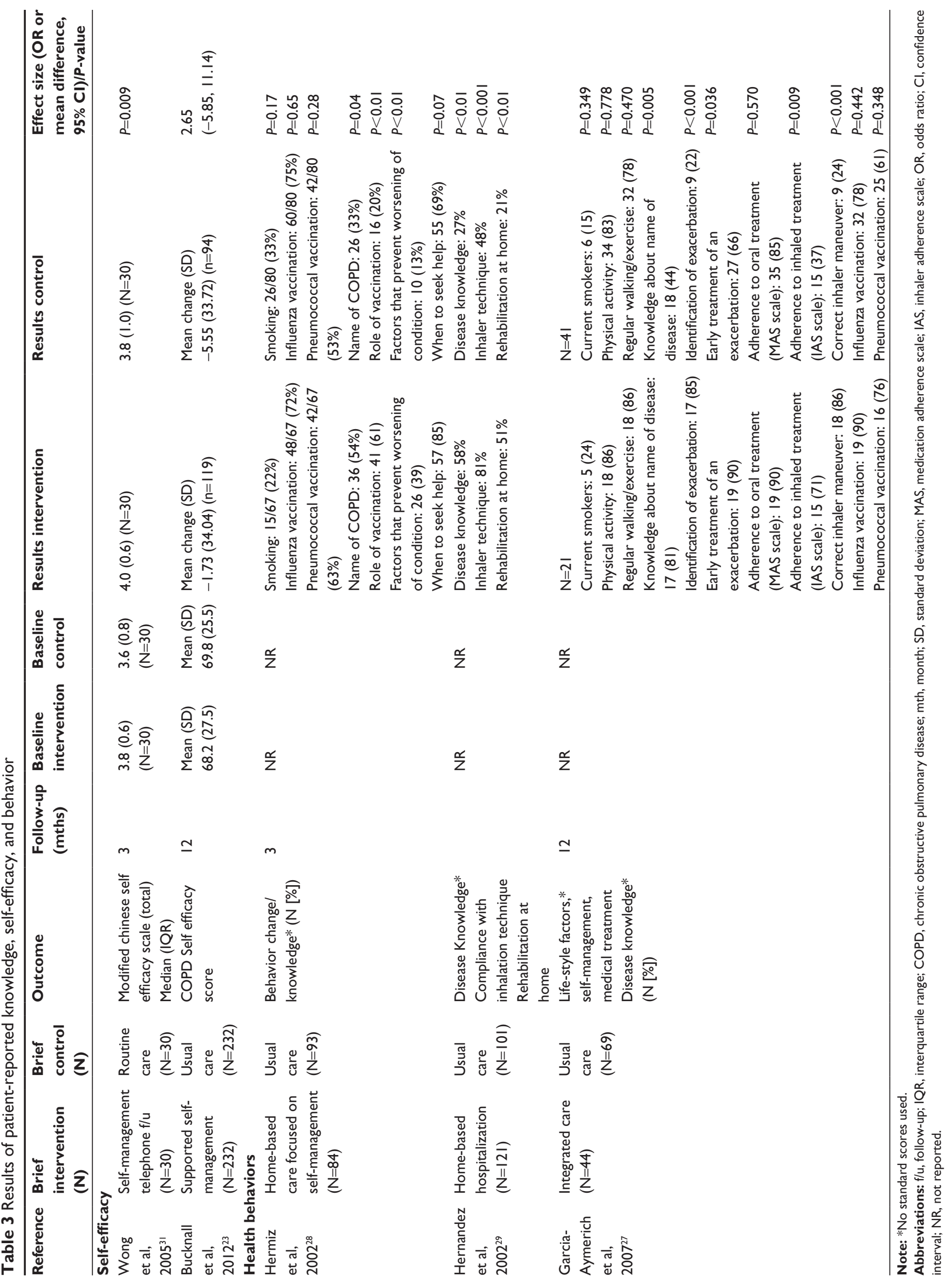


Recently, and particularly since the completion of our searches there has been a number of individual trials and commentaries which question whether patients are actually able to self-manage, or whether it is indeed safe. ${ }^{23,34-36}$ Two of these trials among COPD patients identified a group of successful self-managers in post hoc exploratory analyses which did better, ${ }^{23,34}$ but as only one of these is included in our review, ${ }^{23}$ and no other studies have explored these subgroups, we were unable to examine whether successful self-managers had better outcomes. There is no evidence from our review that the interventions are not safe.

\section{Strengths and weaknesses}

The strength of this review was the rigorous methodological approach with a comprehensive search and selection process which made it unlikely that we would have missed relevant studies.

The main limitation relates to the paucity of evidence and methodological weaknesses of many of the available studies, and the heterogeneous interventions which makes comparisons hard and conclusions difficult to draw. The particular problems with these studies, especially the older ones include generally inadequate reporting of important items. Many studies were small, with data reported only for participants completing the trial and had substantial loss-to-follow-up of more than $30 \%$ in some arms, which is likely to bias all self-reported items and HRQoL in particular. Inability to blind subjects to interventions leaves the self-reported results such as HRQoL open to potential bias. There was a lack of information about the assessment of some outcomes, especially lung function measurements and analyses were often unclear or inappropriate. Outcome measures, eg, admissions, were often reported in different ways, making combined meta-analyses of all the available studies inappropriate. Neither admission nor mortality data were specific to COPD and therefore any effect is most likely to be diluted.

With the limited number of trials it was not possible to assess publication bias, but it is possible that due to the small size of the studies showing positive effects that this is a potential problem.

\section{Implications for research and practice}

It is difficult to recommend any type of self-management support to be provided immediately after discharge as there is no clear evidence of effect across most of the outcomes. This conclusion is in contradiction to the current recommendations for self-management provision in the UK
COPD discharge care bundle, which has been of much interest recently. ${ }^{5}$ However, the lower bounds of the CIs are consistent with positive effects on both mortality and re-admissions, and it may also be the lack of specificity in the cause of these two important outcomes which failed to demonstrate a significant effect.

In this subject area, many of the trials are inadequately reported and suffer from high risk of bias in at least one domain. Any future trials should ensure an adequate standard of reporting, and be conducted to modern standards, with an adequate number of participants and longer follow-up. There should be a clear framework for describing and classifying self-management interventions and their comparators, and clear reporting of outcomes to include self-efficacy, behavior change (including whether patients do self-medicate appropriately during exacerbations) and clinical outcomes, including separate reporting of COPD-related and all-cause admissions. Trials should also report robust effect estimates for the parameters needed for cost-effectiveness analyses. Future studies should also consider that patients may be too ill immediately after an exacerbation (both physically and psychologically) to take up the more rigorous parts of self-management interventions until they are in a more stable state. The difficulty in recruitment and retention in the included studies bears this out. Only one recent trial really addresses current issues and is well-designed and reported. ${ }^{23}$ No difference was found in COPD admissions or death (HR 1.05, 95\% CI 0.80 to 1.38), but a sub-group of patients who became "successful selfmanagers" had better outcomes.

To move forward with this area of research, there should be more in-depth work to explore the needs and views of patients with regard to self-management support after a recent discharge from hospital, with a view to designing novel, perhaps more tailored interventions. We are aware of only limited qualitative research of relevance. ${ }^{14,26}$

Evidence from a Cochrane review of pulmonary rehabilitation trials suggests that a more intensive intervention post-hospitalization including a supervised exercise component reduces hospital admissions (pooled OR 0.22 [95\% CI 0.08 to 0.58$]$ ), over 25 weeks and mortality (OR $0.28 ; 95 \%$ CI 0.10 to 0.84 ) over 107 weeks. ${ }^{16}$ The small exercise study we decided not to pursue formed part of that review. ${ }^{21,22}$ Effects on HRQoL were well above the minimal clinically important difference. However, in common with our review, trials were small, at high risk of bias, and a large proportion of participants did not complete the rehabilitation. There was also significant heterogeneity across many of the outcomes. Interestingly, a more recent robustly performed trial of remotely supervised home rehabilitation showed 
no beneficial effects ${ }^{37}$ and there are well documented problems with low referral, uptake and completion rates. ${ }^{38}$

\section{Conclusion}

General heterogeneity between interventions limits conclusions for many of the outcomes. Self-management support delivered shortly after an acute exacerbation shows an apparent benefit to patients' HRQoL, although this may be overestimated due to high loss-to-follow-up, but there is no evidence of effect on all-cause hospital re-admissions, insufficient information on the effect on respiratory re-admissions, no effect on all-cause mortality, and limited information about the effect on behavior change. The evidence is not currently adequate to support self-management interventions for COPD patients recently after hospital discharge.

\section{Acknowledgments}

The authors thank the following: Simon Stevens for his invaluable administrative support and the people who kindly gave their time to help translate articles: Yumiko Akiya, Dom Barkos, Susan Bayliss, Matthew Blackburn, Yumi Chen, Jennifer Choi, Karin Diaconu, Janine Dretzke, Maxwell Feltham, Ditte Hedegaard, Boris Kysela, Antje Lindenmeyer, Kinga Malottki, Cristina Peñaloza, Amanda Zhang.

\section{Funding}

National Institute for Health Research (NIHR) Health Technology Assessment Programme (10/44/01). Rachel E Jordan was also funded by the NIHR with a post-doctoral research fellowship (pdf/01/2008/023). Kate Jolly is part-funded by the NIHR Collaborations for Leadership in Applied Health Research and Care (CLAHRC) West Midlands. Sally J Singh is part-funded by the Collaboration for Leadership in Applied Health Research and Care East Midlands (CLAHRC EM). The views and opinions expressed therein are those of the authors and do not necessarily reflect those of the HTA programme, NIHR, NHS or the Department of Health. Any errors are the responsibility of the authors.

\section{Publications}

This paper is derived from a larger HTA evidence synthesis report (in press). Part of these data have been presented at the International Primary Care Respiratory Group conference in Stockholm (May 2013) and the British Thoracic Society conference in London (November 2013).

\section{Author contributions}

REJ and KJ led the development of the protocol and directed the review. SB undertook the searching. SM led the selection of studies with assistance from NRH, AMT, DJM, DAF, KJ, REJ and RJ, SM, MJP, NRH, undertook data extraction and evaluation. MJP undertook the statistical analysis with advice from RDR. All authors contributed towards review decision making, data analysis, drafting and revising the manuscript.

\section{Disclosure}

Dr Price reports grants from UK Medical Research Council, during the conduct of the study; Dr Turner reports grants from University of Birmingham/NIHR, during the conduct of the study; Dr Jordan, Prof Jolly, Prof Adab, Prof Fitzmaurice, and Dr Moore report grants from the NIHR, during the conduct of the study; Prof Singh reports that the University Hospitals of Leicester NHS trust holds the intellectual property for a self management manual for COPD and reports a British Lung Foundation grant for RCT of self-management support post admission. The authors report no other conflicts of interest.

\section{References}

1. Global Initiative for Chronic Obstructive Lung Disease [homepage on the Internet]. Global Strategy for the Diagnosis, Management and Prevention of COPD. Global Initiative for Chronic Obstructive Lung Disease (GOLD) 2014. Available from: http://www.goldcopd.org/. Accessed December 8, 2014.

2. Donaldson GC, Wedzicha JA. COPD exacerbations 1: Epidemiology. Thorax. 2006;61(2):164-168.

3. Zwerink M, Brusse-Keizer M, van der Valk PD, et al. Self management for patients with chronic obstructive pulmonary disease. Cochrane Database Syst Rev. 2014;3:CD002990.

4. Walters JA, Turnock AC, Walters EH, Wood-Baker R. Action plans with limited patient education only for exacerbations of chronic obstructive pulmonary disease. Cochrane Database Syst Rev. 2010; (5):CD005074.

5. Hopkinson NS, Englebretsen C, Cooley N, et al. Designing and implementing a COPD discharge care bundle. Thorax. 2011;67(1):90-92.

6. Disler RT, Gallagher RD, Davidson PM. Factors influencing selfmanagement in chronic obstructive pulmonary disease: an integrative review. Int J Nurs Stud. 2012;49(2):230-242.

7. Bourbeau J, van der Palen J. Promoting effective self-management programmes to improve COPD. Eur Respir J. 2009;33(3):461-463.

8. Nici L, Donner C, Wouters E, et al. American Thoracic Society/ European Respiratory Society statement on pulmonary rehabilitation. Am J Respir Crit Care Med. 2006;173(12):1390-1413.

9. Effing TW, Bourbeau J, Vercoulen J, et al. Self-management programmes for COPD: moving forward. Chron Respir Dis. 2012;9(1): $27-35$.

10. Williams V, Hardinge M, Ryan S, Farmer A. Patients' experience of identifying and managing exacerbations in COPD: a qualitative study. NPJ Prim Care Respir Med. 2014;24:14062.

11. Bourbeau J, Julien M, Maltais F, et al. Reduction of hospital utilization in patients with chronic obstructive pulmonary disease: a disease-specific self-management intervention. Arch Intern Med. 2003;163(5):585-591.

12. National Institute for Health and Clinical Excellence [homepage on the Internet]. Chronic obstructive pulmonary disease: management of chronic obstructive pulmonary disease in adults in primary and secondary care. NICE clinical guideline 101; 2010. London: National Institute for Health and Clinical Excellence. Available from: www.guidance. nice.org.uk/cg101. Accessed December 8, 2014. 
13. Wagg K. Unravelling self-management for COPD: what next? Chron Respir Dis. 2012;9(1):5-7.

14. Harrison SL, Apps L, Singh SJ, Steiner MC, Morgan MDL, Robertson M. Consumed by breathing - a critical interpretive meta-synthesis of the qualitative literature. Chronic Illn. 2014;10(1):31-49.

15. Barlow J, Wright C, Sheasby J, Turner A, Hainsworth J. Self-management approaches for people with chronic conditions: a review. Patient Educ Couns. 2010;48(2):177-187.

16. Puhan MA, Gimeno-Santos E, Scharplatz M, Troosters T, Walters EH, Steurer J. Pulmonary rehabilitation following exacerbations of chronic obstructive pulmonary disease. Cochrane Database Syst Rev. 2011; (10):CD005305.

17. Higgins JP, Green S, editors. Chapter 8: Assessing risk of bias in included studies. In: Cochrane Handbook for Systematic Reviews of Interventions Version 5.0.2. 2009:187-241.

18. Parmar MK, Torri V, Stewart L. Extracting summary statistics to perform meta-analyses of the published literature for survival endpoints. Stat Med. 1998;17(24):2815-2834.

19. Perneger TV. Estimating the relative hazard by the ratio of logarithms of event-free proportions. Contemp Clin Trials. 2008;29(5):762-766.

20. Lee DT, Lee IF, Mackenzie AE, Ho RN. Effects of a care protocol on care outcomes in older nursing home patients with chronic obstructive pulmonary disease. J Am Geriatr Soc. 2002;50(5):870-876.

21. Behnke M, Taube C, Kirsten D, et al. Home-based exercise is capable of preserving hospital-based improvements in severe chronic obstructive pulmonary disease. Respir Med. 2000;94(12):1184-1191.

22. Behnke M, Jorres RA, Kirsten D, Magnussen H. Clinical benefits of a combined hospital and home-based exercise programme over 18 months in patients with severe COPD. Monaldi Arch Chest Dis. 2003;59(1):44-51.

23. Bucknall CE, Miller G, Lloyd SM, et al. Glasgow supported selfmanagement trial (GSuST) for patients with moderate to severe COPD: randomised controlled trial. BMJ. 2012;344:e1060.

24. Casas A, Troosters T, Garcia-Aymerich J, et al. Integrated care prevents hospitalisations for exacerbations in COPD patients. Eur Respir J. 2006;28(1):123-130.

25. Dheda K, Crawford A, Hagan G, Roberts CM. Implementation of British Thoracic Society guidelines for acute exacerbation of chronic obstructive pulmonary disease: Impact on quality of life. Postgrad Med J. 2004;80(941):169-171.

26. Egan E, Clavarino A, Burridge L, Teuwen M, White E. A randomized control trial of nursing-based case management for patients with chronic obstructive pulmonary disease. Lippincotts Case Manag. 2002;7(5):170-179.
27. Garcia-Aymerich J, Hernandez C, Alonso A, et al. Effects of an integrated care intervention on risk factors of COPD readmission. Respir Med. 2007;101(7):1462-1469.

28. Hermiz O, Comino E, Marks G, et al. Randomised controlled trial of home based care of patients with chronic obstructive pulmonary disease. BMJ. 2002;325(7370):938.

29. Hernandez C, Casas A, Escarrabill J, et al. Home hospitalisation of exacerbated chronic obstructive pulmonary disease patients. Eur Respir J. 2003;21(1):58-67.

30. Kwok T, Lum CM, Chan HS, et al. A randomized, controlled trial of an intensive community nurse-supported discharge program in preventing hospital readmissions of older patients with chronic lung disease. $J \mathrm{Am}$ Geriatr Soc. 2004;52(8):1240-1246.

31. Wong KW, Wong FK, Chan MF. Effects of nurse-initiated telephone follow-up on self-efficacy among patients with chronic obstructive pulmonary disease. $J$ Adv Nurs. 2005;49(2):210-222.

32. Jones PW. Interpreting thresholds for a clinically significant change in health status in asthma and COPD. Eur Respir $J$ 2002;19(3):398-404.

33. Kruis AL, Smidt N, Assendelft WJ, et al. Integrated disease management interventions for patients with chronic obstructive pulmonary disease. Cochrane Database Syst Rev. 2013;10:CD009437.

34. Bischoff EW, Akkermans R, Bourbeau J, et al. Comprehensive self management and routine monitoring in chronic obstructive pulmonary disease patients in general practice: randomised controlled trial. $B M J$. 2012;345:e7642.

35. Fan VS, Gaziano JM, Lew R, et al. A comprehensive care management program to prevent chronic obstructive pulmonary disease hospitalizations. A randomized, controlled trial. Ann Intern Med. 2012;156(10):673-683

36. Wedzicha JA, Vestbo J. Can patients with COPD self-manage? Lancet. 2012;380(9842):624-625

37. Greening NJ, Williams JEA, Hussain SF, et al. An early rehabilitation intervention to enhance recovery during hospital admission for an exacerbation of chronic respiratory disease: randomised controlled trial. BMJ. 2014;349:g4315.

38. Jones SE, Green SA, Clark AL, et al. Pulmonary rehabilitation following hospitalisation for acute exacerbation of COPD: referrals, uptake and adherence. Thorax. 2014;69(2):181-182.
International Journal of COPD

\section{Publish your work in this journal}

The International Journal of COPD is an international, peer-reviewed journal of therapeutics and pharmacology focusing on concise rapid reporting of clinical studies and reviews in COPD. Special focus is given to the pathophysiological processes underlying the disease, intervention programs, patient focused education, and self management protocols.

\section{Dovepress}

This journal is indexed on PubMed Central, MedLine and CAS. The manuscript management system is completely online and includes a very quick and fair peer-review system, which is all easy to use. Visit http://www.dovepress.com/testimonials.php to read real quotes from published authors. 